The Astrophysical Journal, 173 :L71-L74, 1972 April 15

(C) 1972. The University of Chicago. All rights reserved. Printed in U.S.A.

\title{
INFRARED DIAMETER OF IRC+10216 DETERMINED FROM LUNAR OCCULTATIONS
}

\author{
R. I. Toombs, ${ }^{*} \dagger$ E. E. Becklin, ${ }^{*} \ddagger$ J. A. Frogel, ${ }^{*}$ S. K. LaW, \\ F. C. Porter, * and J. A. Westphal $\neq \|$ \\ Received 1972 February 9
}

\section{ABSTRACT}

Lunar occultations of IRC +10216 have been used to determine the size of the regions emitting 2.2-, 3.5-, 4.8-, and 10- $\mu$ radiation. The results are interpreted as the first direct measurement of the physical size of a dust shell surrounding a late-type star.

During the winter of 1970-1971, two lunar occultations of the bright infrared object IRC+10216 (Becklin et al. 1969) were observed from sites in California and Arizona. These observations have resulted in determinations of the angular size of this object at $2.2 \mu(\Delta \lambda=0.4 \mu), 3.5 \mu(\Delta \lambda=0.6 \mu), 4.8 \mu(\Delta \lambda=0.6 \mu)$, and $10 \mu(\Delta \lambda=5 \mu)$.

The 3.5-, 4.8-, and 10- $\mu$ observations were made on 1970 November 20 on the 24- and 60-inch $(61$ and $152 \mathrm{~cm})$ telescopes at Mount Wilson Observatory and the 200-inch $(508 \mathrm{~cm})$ Hale Telescope at Palomar Observatory, respectively. The $2.2-\mu$ observation was made on 1971 January 14 on the 60 -inch Tillinghast telescope at Mount Hopkins Observatory. Conventional infrared photometers (Becklin and Neugebauer 1968) and data systems were modified to allow chopping frequencies higher than $25 \mathrm{~Hz}$ and time resolutions shorter than $50 \mathrm{~ms}$. Table 1 summarizes the relevant parameters of these observations; because IRC +10216 is variable, the magnitudes at the time of the occultations are also given.

The four occultation curves obtained as IRC +10216 emerged from behind the dark limb of the Moon are shown in figure 1. For comparison, figure 1 also shows the data from the occultation of the M4 star HD 76830 observed at $2.2 \mu$ on the 24-inch telescope on 1970 November 19. All sets of data were normalized to uniform spatial resolution by taking a running average over $100-\mathrm{ms}$ time intervals; the time coordinate can be converted to angular distance in the sky by using the apparent rate and direction of lunar motion. For the observations of IRC $+10216,1$ second of time in figure 1 corresponds to 0 ". $34 \pm 0$ ".01 in the sky; for HD 76830, 1 second of time corresponds to 0 ". $29 \pm 0$ ".2. The $10-\mu$ measurement of IRC +10216 was affected by the fact that the image was only two-thirds within the $10^{\prime \prime}$ aperture upon reappearance from behind the Moon while the seeing disk was greater than $5^{\prime \prime}$. At $4.8 \mu$ a series of recurrent noise spikes did not allow the complete determination of the reappearance curve.

An accurate measurement of the time of reappearance at $2.2 \mu$ was made at Mount Hopkins, allowing a determination of the position of the infrared source along the path of the Moon which was in a southeast direction. The position of the optical image (Becklin et al. 1969) relative to the infrared position is indicated in figure 1.

The data in figure 1 clearly show that IRC +10216 is extended. Several models for

* Division of Physics, Mathematics, and Astronomy, California Institute of Technology.

$\dagger$ Present address: Jet Propulsion Laboratory, California Institute of Technology.

$\ddagger$ Staff Associate, Hale Observatories, California Institute of Technology, Carnegie Institution of Washington.

$\S$ Present address: Harvard College Observatory.

|| Division of Geological and Planetary Sciences, California Institute of Technology.

$\mathrm{L} 71$ 
TABLE 1

\begin{tabular}{|c|c|c|c|c|}
\hline Object & Date (UT) & Telescope (inches) & $\lambda_{\text {eff }}(\mu)$ & Magnitude* \\
\hline IRC+10216. . & $1971 \operatorname{Jan} 14$ & 60 Mount Hopkins & 2.2 & 0.1 \\
\hline IRC $+10216 \ldots$ & 1970 Nov 20 & 24 Mount Wilson & 3.5 & -2.6 \\
\hline IRC $+10216 \ldots$ & 1970 Nov 20 & 60 Mount Wilson & 4.8 & -4.4 \\
\hline IRC $+10216 \ldots .$. & 1970 Nov 20 & 200 Palomar & 10.2 & -7.2 \\
\hline HD $76830 \ldots \ldots \ldots$ & 1970 Nov 19 & 24 Mount Wilson & 2.2 & 1.2 \\
\hline
\end{tabular}

* On 1970 November 20 the $2.2-\mu$ magnitude of IRC +10216 was +1.0 .

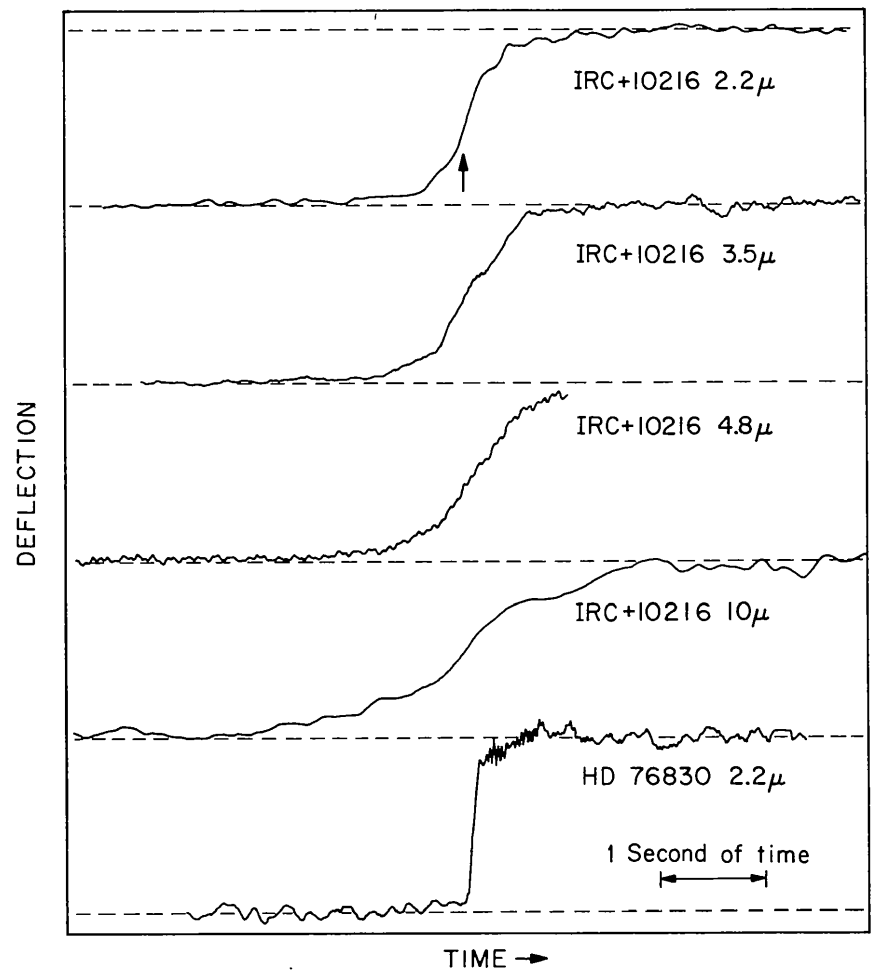

FIG. 1.-The normalized reappearance curves of IRC+10216 at $2.2,3.5,4.8$, and $10 \mu$ are shown. One second of time corresponds to $0^{\prime \prime} 34 \pm 0$. 01 . The dashed lines represent the average baselines approximately $10 \mathrm{~s}$ before and $10 \mathrm{~s}$ after the occultation. For all data a running average over 100-ms intervals has been made to provide equal time resolution. The $4.8-\mu$ curve has not been completely determined because of a series of recurrent noise spikes. An occultation of the M4 star HD 76830 at $2.2 \mu$ is shown for comparison; the rate is $0.29 \pm 0$ ".02 per second of time. The arrow shown on the $2.2-\mu$ curve of IRC +10216 corresponds to the position of the optical image identified by Becklin et al. (1969). The quoted uncertainty in the optical position is \pm 1 second of time.

the spatial distribution of infrared radiation have been fitted to the occultation curves; in general, a good fit to the observations is obtained by assuming disks with uniform surface brightness and diameters of $0.36 \pm 0$. 02,0 . $44 \pm 0$ ".02, and $0.44 \pm 0$ ".02 at $2.2,3.5$, and $4.8 \mu$ respectively. Specifically, at $2.2 \mu$ not more than 20 percent of the radiation can come from a central unresolved star. In addition to the 0.4 disk, it is necessary, at all wavelengths, to add a second, more extended component having a diameter of $2 "$; the flux in this component contains 10-20 percent of the flux in the central disk at wavelengths shorter than $5 \mu$. Although the data at $10 \mu$ are of a poorer quality, 
it appears that approximately 50 percent of the radiation comes from the central 0 ". 4 diameter source and 50 percent from the outer 2 " region.

If the magnitudes given in table 1 are used, then the central 0.4 component has a brightness temperature of approximately $600^{\circ} \mathrm{K}$, which is in good agreement with the average color temperature at 1.2, 1.6, 3.5, and $4.8 \mu$ (Becklin et al. 1969). This would indicate that the source radiates like a blackbody at these wavelengths. Dust shells have been hypothesized to explain the observed infrared energy distributions of many classes of stars (Johnson, Low, and Steinmetz 1965; Gillett, Low, and Stein 1968; Hyland et al. 1969; Woolf and Ney 1969; Low et al. 1970). Although the present observations cannot be used to determine the constitution of the radiating material, they clearly indicate that at least in the case of IRC+10216, most of the observed infrared flux results from an optically thick extended source.

Spectra in the 1- $\mu$ region by Lockwood (1970), Miller (1970), and Herbig and Zappala (1970) indicate the presence of a central late-type carbon star. This result is consistent with the present observations if the $1-\mu$ radiation is scattered by the particles in the optically thick inner shell; 20 percent polarization at $1 \mu$ measured by Shawl and Zellner (1970) tends to confirm that this is correct. Thermal reemission from the shell near $1 \mu$ does not appear to be sufficient to mask the scattered absorption spectrum. IRC +10216 thus probably represents an extreme example of the sequence of carbon stars surrounded by dust shells (Frogel and Hyland 1972).

As mentioned above, the occultation observations indicate the presence of a second larger shell $2^{\prime \prime}$ in diameter. Two estimates of the temperature of this outer shell can be made. From the $4.8-$ to $10-\mu$ color, the calculated temperature is about $350^{\circ} \mathrm{K}$. A second estimate can be obtained from the 10- and 20- $\mu$ photometry ${ }^{1}$ of Becklin et al. (1969). The measured 10 - and $20-\mu$ flux exceeds, by a factor of about 2 , that expected from a $650^{\circ} \mathrm{K}$ blackbody fit through the data at $\lambda<5 \mu$. The results of the present occultation observations would indicate that the excess $10-\mu$ flux originates from the outer 2 " shell. If this interpretation of the excess is also correct at $20 \mu$, then the color temperature of the outer shell is about $400^{\circ} \mathrm{K}$. In contrast, the $10-\mu$ brightness temperature of the $2^{\prime \prime}$ diameter disk is about $270^{\circ} \mathrm{K}$.

The luminosity in the $2^{\prime \prime}$ shell is about 30 percent of the total luminosity. If one adopts a temperature of $375^{\circ} \mathrm{K}$ and mean diameter of $2^{\prime \prime}$ for the shell, the average $10-\mu$ optical depth is about 0.2 . Both these values indicate that the outer shell is optically thin, in agreement with the fact that the inner 0 ". 4 shell is clearly visible through the outer shell at $2.2,3.5$, and $4.8 \mu$. In contrast to the temperatures estimated above, the thermal equilibrium temperature of gray particles in the outer $2^{\prime \prime}$ shell would be about $200^{\circ} \mathrm{K}$. Such a low temperature not only is inconsistent with photometric results discussed above but is lower than the observed $10-\mu$ brightness temperature. It thus appears that the particles in the $2^{\prime \prime}$ shell are nongray. The large $2.2-\mu$ flux seen in the outer shell does not necessarily result from thermal emission at $375^{\circ} \mathrm{K}$; rather, it is more likely that it is attributable to scattering from the inner dust shell.

To summarize, the schematic model which is deduced for IRC +10216 is that of a late-type carbon star surrounded by a dust shell which scatters some of the starlight, but probably absorbs and thermally reemits a majority of it. This dust shell may be represented by two components: a bright, optically thick central component with a characteristic diameter and temperature of 0.4 and $600^{\circ} \mathrm{K}$, respectively, and a less luminous optically thin outer component having a characteristic diameter of $2^{\prime \prime}$ and a temperature around $375^{\circ} \mathrm{K}$.

\footnotetext{
${ }^{1}$ Recalibration of the Caltech 10 - and 20- $\mu$ magnitude system indicates that the $10-\mu$ magnitude and flux given in table 1 of Becklin et al. (1969) should read $-7.7 \mathrm{mag}$ and $14.0 \times 10^{-14} \mathrm{~W} \mathrm{~cm}^{-2} \mu^{-1}$ while the $20-\mu$ magnitude and flux should read -8.8 mag and $2.4 \times 10^{-14} \mathrm{~W} \mathrm{~cm}^{-2} \mu^{-1}$ (Becklin, Hansen, and Neugebauer 1972).
} 
We would like to thank Professor G. Neugebauer and members of the Caltech infrared group for their support of this work. For help in making the observations we would like to thank G. Clough, J. Mosher, T. Gregory, G. Tuton, B. Van't Sant, and E. Horine. D. Espenschield of the U.S. Naval Observatory kindly provided information on the lunar rate and position. E. E. B. and J. A. F. would like to thank the Smithsonian Astrophysical Observatory for use of the 60-inch Mount Hopkins telescope. This work was supported in part by National Aeronautics and Space Administration grants NGL 05-002-207 and NGL 05-002-007 to the California Institute of Technology. J. A. F. was supported in part by Harvard College Observatory.

\section{REFERENCES}

Becklin, E. E., Frogel, J. A., Hyland, A. R., Kristian, J., and Neugebauer, G. 1969, A p. J. (Letters), 158, L133.

Becklin, E. E., Hansen, O. V., and Neugebauer, G. 1972 (in preparation).

Becklin, E. E., and Neugebauer, G. 1968, $A$ p. J., 151, 145.

Frogel, J. A., and Hyland, A. R. 1972, Proceedings of the Seventeenth International Astrophysical Symposium, Liège (in press).

Gillett, F. C., Low, F. J., and Stein, W. A. 1968, A p. J., 154, 677.

Herbig, G. H., and Zappala, R. R. 1970, A p. J. (Letters), 162, L15.

Hyland, A. R., Becklin, E. E., Neugebauer, G., and Wallerstein, G. 1969, A p. J., 158, 619.

Johnson, H. L., Low, F. J., and Steinmetz, D. 1965, A p. J., 142, 808.

Lockwood, G. W. 1970, A p. J. (Letters), 160, L47.

Low, F. J., Johnson, H. L., Kleinmann, D. E., Latham, A. S., and Geisel, S. L. 1970, A p. J., 160, 531.

Miller, J. S. 1970, Ap.J. (Letters), 161, L95.

Shawl, S. J., and Zellner, B. 1970, A p. J. (Letters), 162, L19.

Woolf, N. J., and Ney, E. P. 1969, A p. J. (Letters), 155, L181. 